\title{
A suitable marking method to achieve lateral margin negative in endoscopic submucosal dissection for undifferentiated-type early gastric cancer
}

\section{(ㄷ)(우우}

\author{
Authors

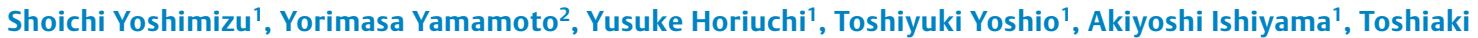 \\ Hirasawa ${ }^{1}$, Tomohiro Tsuchida ${ }^{1}$, Junko Fujisaki ${ }^{1}$
}

Institutions

1 Department of Gastroenterology, Cancer Institute Hospital, Tokyo, Japan

2 Division of Gastroenterology, Showa University Fujigaoka Hospital, Endoscopy Center, Yokohama, Japan

submitted 18.7.2018

accepted after revision 21.11.2018

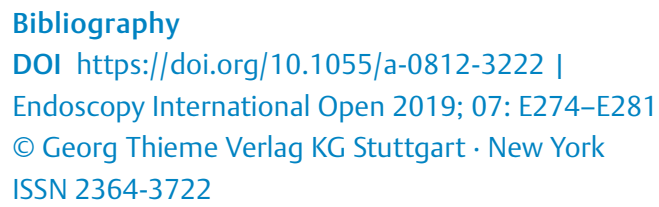

Corresponding author

Shoichi Yoshimizu, Division of Gastroenterology, Cancer Institute Hospital, Japanese Foundation for Cancer Research, 3-10-6 Ariake, Koto-ku, Tokyo 135-8550, Japan Fax: +010-81-3-3520-0141

shoichi.yoshimizu@jfcr.or.jp

\section{ABSTRACT}

Background and study aims Delineating undifferentiated-type early gastric cancer (UD-type EGC) from noncancerous areas is difficult. Therefore, the lateral margin negative $(\mathrm{LM}-)$ resection rate of endoscopic submucosal dissec- tion (ESD) is lower for UD-type EGC than for differentiatedtype EGC. This study aimed to retrospectively evaluate the effectiveness of the marking methods with circumferential biopsies in ESD for UD-type EGC.

Patients and methods We analyzed the clinical outcomes of ESD in 127 patients with UD-type EGC between April 2013 and 2017. We performed diagnostic delineation of cancerous areas using magnifying endoscopy with narrowband imaging, and four or more circumferential biopsies approximately $5 \mathrm{~mm}$ apart from the estimated lesion border were obtained to confirm noncancerous areas. The markings were placed on the circumferential biopsy scars, and a mucosal incision line was made outside the markings. Results Median size of the tumors and ESD specimens was 12 and $35 \mathrm{~mm}$, respectively. En-bloc resection rate was $100 \%(127 / 127)$, and LM- and curative resection rates were $97.6 \%(124 / 127)$ and $80.3 \%(102 / 127)$, respectively. Circumferential biopsy in preoperative esophagogastroduodenoscopy has successfully identified the misdiagnosis of cancerous areas of four patients (3.2\%), with three (2.4\%) achieving $\mathrm{LM}$ - resection. $\mathrm{LM}+$ resection was pathologically identified in three patients $(2.4 \%)$, with all undergoing non-curative resection due to $>20$-mm tumor. The proportion of patients with the shortest distance $\geq 5 \mathrm{~mm}$ from the lesion edge to the specimen edge was $88.2 \%$ (112/127). Conclusion Our marking methods with circumferential biopsies may reduce $\mathrm{LM}+$ resections in ESD for UD-type EGC.

\section{Introduction}

Gastric cancer is a prevalent malignancy in East Asian countries [1]. Technological advancements in endoscopic submucosal dissection (ESD) have prompted widespread use of endoscopic resection (ER) for early gastric cancer (EGC), not only in Japan but also in other countries. According to reports of Hirasawa et al. [2], undifferentiated-type early gastric cancer (UD-type EGC) is one of the expanded indications for ESD in the Japanese Gastric Cancer Association Guideline [3] as long as it is an intramucosal lesion measuring $\leq 20 \mathrm{~mm}$, ulcer negative, and without lympho-vascular invasion, thereby reflecting the negligible risk of lymph node metastasis. Thus, inclusion of UD-type EGC in expanded indications for ESD is currently widely accepted in Japan.

However, delineating the cancerous areas is sometimes difficult when UD-type EGC is not exposed on the mucosal surface and, instead, has progressed laterally along the proliferative zone within the middle layer of the mucosa [4]. As a result, the lateral margin negative $\left(\mathrm{LM}^{-}\right)$resection rate for UD-type EGC was reported as $72.7 \%$ to $94.8 \%$ [5-9], which is lower than 
the result of differentiated-type early gastric cancer (D-type EGC) $[10-12]$.

Regarding the ESD procedure, the markings were commonly placed entirely around the lesion before the mucosal incision. Generally, for ESD of D-type EGC, the markings are placed 2 to $3 \mathrm{~mm}$ apart or more from the estimated border of the lesion area [10]. For UD-type EGC, some previous studies reported that the markings were placed 5 to $10 \mathrm{~mm}$ apart from the estimated border of the lesion area [5,7]. However, the details of the suitable marking method are still not clarified.

We had reported that the magnifying endoscopy with narrow-band imaging (M-NBI) diagnosis and circumferential biopsies, which confirm the non-neoplastic mucosa in preoperative esophagogastroduodenoscopy (EGD), are needed to achieve LM-resection in ESD for UD-type EGC [5]. Therefore, we necessarily perform M-NBI diagnosis and circumferential biopsies for ESD cases of UD-type EGC. In this study, we aimed to retrospectively evaluate the effectiveness of the marking methods with circumferential biopsies in ESD for UD-type EGC.

\section{Patients and methods}

\section{Patients}

In our institution, we carried out ER for EGC in 1,731 patients between April 2013 and 2017. Of these patients, 1,570 were preoperatively diagnosed with D-type EGC, whereas 161 were preoperatively diagnosed with UD-type EGC. Of the 161 patients, we excluded 14 with differentiated dominant-type EGC on ESD specimens, seven with prior gastrectomy and reconstructive surgery involving the stomach for esophageal cancer, four in whom circumferential biopsies were not performed in preoperative EGD, three with lesions located near ulcer scar or anastomosis, three in whom the tumor edge was in contact with the esophagogastric junction or pyloric ring, two with simultaneous multiple EGCs, and one who underwent endoscopic mucosal resection. We analyzed clinical outcomes of ESD in 127 patients with UD-type EGC who fulfilled the expanded indications for ESD ( $\triangleright$ Fig. 1). Written informed consent was obtained from all patients prior to undergoing the procedure. This study was approved by the Institutional Review Board of Cancer Institutional Hospital (IRB No. 2017 -1113).

\section{Strategy of preoperative EGD}

Preoperative EGD was performed in all patients. We mainly used the LUCERA-ELITE system (Evis Lucera Elite System; Olympus Medical Systems, Tokyo, Japan) and GIF-H260Z or GIFH290Z endoscope (Olympus Medical Systems). We carried out diagnostic delineation of cancerous areas using M-NBI and dye spraying endoscopy with application of indigo carmine after white light imaging (WLI) observation. In the M-NBI diagnosis, we also carried out diagnostic delineation of cancerous areas using expansion of the intervening parts, which enhances the diagnostic capability [13]. Following the M-NBI diagnosis, circumferential biopsies approximately $5 \mathrm{~mm}$ apart from the estimated border of lesion were obtained at even intervals. Four circumferential biopsies were standardized, but if the interval of the circumferential biopsies was widened or the border of le-
Patients carried out ER for EGC in Cancer Institute Hospital between April 2013 and 2017 ( $n=1,731$ )

Excluded $(\mathrm{n}=1,570)$

1,570 who carried out ER for preoperatively diagnosed with differentiated type EGC

Patients carried out ER for preoperatively diagnosed with UD-type EGC between April 2013 and 2017 $(n=161)$

Excluded $(n=34)$

- 14 with differentiated dominant-type on ESD specimen

- 7 with prior gastrectomy and reconstructive surgery involving the stomach for esophageal cancer

- 4 in whom circumferential biopsies were not performed before ESD

- 3 with lesions located near ulcer scar or anastomosis

- 3 in whom the tumor edge was in contact with EGJ or pyloric ring

- 2 with simultaneous multiple cancers

- 1 who underwent EMR

Clinical outcomes of ESD in patients with UD-type EGC were analyzed

- 110 with signet ring cell carcinoma

- 17 with poorly differentiated adenocarcinoma

$(\mathrm{n}=127)$

- Fig. 1 Flowchart of inclusion of the patients with undifferentiated-type early gastric cancer. ER, endoscopic resection; EGC, early gastric cancer; UD-type EGC, undifferentiated type early gastric cancer; ESD, endoscopic submucosal dissection; EMR, endoscopic mucosal resection; EG], esophagogastric junction.

sion was unclear, five or six circumferential biopsies were performed. If the result of the circumferential biopsy was cancerpositive, even in only one of the specimens, we performed a secondary preoperative EGD to confirm whether the circumferential biopsies were cancer-negative.

\section{ESD procedure}

ESD was carried out under the supervision of an expert endoscopist certified by the Japan Gastroenterological Endoscopy Society with the patient anesthetized with midazolam and pethidine hydrochloride. We performed ESD using the insulated-tip (IT) knife2 (Olympus Medical Systems) as the primary resection device, and ERBE VIO 300 D (Erbe, Tubingen, Germany) or ESG100 (Olympus Medical Systems) as the electrosurgical generator. To lift the mucosa, $0.4 \%$ sodium hyaluronate solution (Mucoup, Boston Scientific Co., Tokyo, Japan and Seikagaku Co., Tokyo, Japan) was then injected into the submucosal layer. Markings were placed on the circumferential biopsy scars using the 

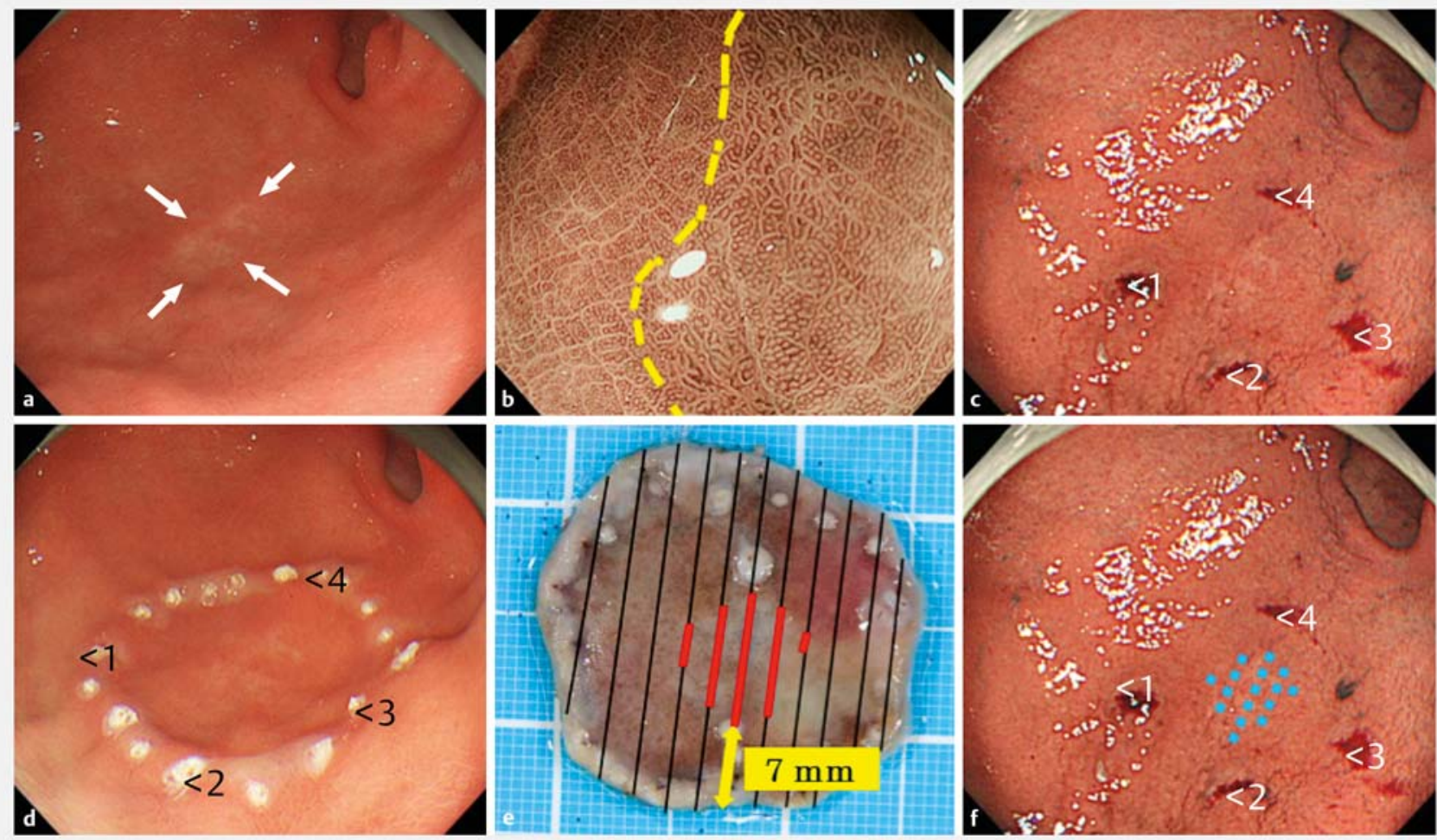

\begin{abstract}
- Fig. 2 A case of undifferentiated-type early gastric cancer with successful lateral margin negative resection in a 56-year-old man. a In preoperative esophagogastroduodenoscopy, a discolored lesion is located on the greater curvature of the antrum (white arrow). b In M-NBI diagnosis, a diagnostic delineation of cancerous areas (yellow dotted line) using expansion of the intervening parts is performed. c Four circumferential biopsies (numbers 1 to 4 ) approximately $5 \mathrm{~mm}$ apart from the estimated border of the lesion are obtained, which are identified as cancernegative samples. $\mathbf{d}$ The markings are placed on the circumferential biopsy scars and ESD is performed. e Histological mapping: the ESD specimen is excised as indicated by the black lines. The area of the lesion itself is represented by the red lines. The shortest distance from the lesion edge to the specimen edge is $7 \mathrm{~mm}$. It is diagnosed as successful LM- resection. Pathological findings: ESD specimen size, $35 \times 33 \mathrm{~mm}$; tumor size, $12 \times 12 \mathrm{~mm}$; and type 0 -IIb, signet ring cell carcinoma, UL-, M, ly0, v0, LM-,VM-. $\mathbf{f}$ On retrospective consideration, the area of the lesion itself is depicted by the blue-dotted line. M-NBI, magnifying endoscopy with narrow-band imaging; ESD, endoscopic submucosal dissection; LM, lateral margin; VM, vertical margin.
\end{abstract}

GIF-H260Z or GIF-H290Z endoscope and an argon plasma coagulator (APC) probe or snare tip. When circumferential biopsy scars were not recognizable, the preoperative EGD images of the biopsies were considered. Subsequently, the markings were placed at least $5 \mathrm{~mm}$ apart from the estimated border of the lesion area. A mucosal incision line was made outside the markings. The mucosal incisions and submucosal dissections were mainly carried out using GIF-Q260) (Olympus Medical Systems), which has a water-jet function as well. After lesion dissection, a preventive hemostatic procedure to coagulate vessels on the artificial ulcers was immediately performed using hemostatic forceps. $>$ Fig. 2 shows an example of a marking method.

\section{Pathological diagnosis}

Pathological findings of ESD specimens were evaluated using version 14 of the Japanese Classification of Gastric Carcinoma [14]. All ESD specimens were sectioned into 2-mm slices and evaluated through histopathological examinations. The Japanese classification system categorizes histological types of gastric carcinoma into the following groups: differentiated and un- differentiated. The differentiated group consists of well-differentiated carcinoma, moderately differentiated carcinoma, and papillary adenocarcinoma, whereas the undifferentiated group consists of poorly differentiated adenocarcinoma (PDAC) and signet ring cell carcinoma (SRC). LM was considered negative if no cancer cells were present within $2 \mathrm{~mm}$ from the specimen edge.

\section{Therapeutic outcome parameters}

We evaluated characteristics of the patients with UD-type EGC and their lesions in terms of the following parameters: age, sex, location, gross type, tumor size, circumferential biopsies, and histology. Definitions used for the evaluation of ESD therapeutic outcomes were as follows: en-bloc resection was defined as the successful resection of a lesion in one piece, irrespective of the pathological findings; $R 0$ resection was en-bloc resection with both the lateral and vertical margins being negative for cancer cells; and curative resection as resection that satisfied the expanded indications for ESD. ESD operation time was defined as the duration from endoscope insertion to its removal. 
- Table 1 Characteristics of the patients and lesions with undifferentiated-type early gastric cancer (UD-type EGC).

\begin{tabular}{|l|c|}
\hline & UD-type EGC (n=127) \\
\hline Age, median (range), year & $56(26-80)$ \\
\hline Sex, n (\%) Male/female & $74(58.3) / 53(41.7)$ \\
\hline Gross type, n (\%) 0-II a/0-II b/0-II c & $7(5.5) / 26(20.5) / 94(74.0)$ \\
\hline Location, n (\%) Upper third/middle third/lower third & $1(0.8) / 86(67.7) / 40(31.5)$ \\
\hline Tumor diameter (preoperative diagnosis), median (range), mm & $13(3-20)$ \\
\hline Circumferential location, n (\%) Ant/Pos/Les/Gra & $25(19.7) / 27(21.2) / 38(29.9) / 27(21.2)$ \\
\hline Number of circumferential biopsies, n (\%) Four/Five/Six & $110(86.6) / 13(10.2) / 4(3.2)$ \\
\hline Circumferential biopsy cancer positive, n (\%) & $4(3.2)$ \\
\hline Histology (preoperative biopsy), n (\%) SRC/PDAC & $108(85.0) / 19(15.0)$ \\
\hline $\begin{array}{l}\text { Ant, Anterior wall; Pos, Posterior wall; Les, Lesser curvature; Gra, Grater curvature } \\
\text { SRC, signet ring cell carcinoma; PDAC, poorly differentiated adenocarcinoma }\end{array}$ \\
\hline
\end{tabular}

We, then, investigated the shortest distance from the lesion edge to the specimen edge. The pathologist serially sectioned the post-ESD specimens at 2-mm intervals to histologically estimate the area of the lesions, which was mapped on a photograph for measurement of the shortest distance ( $\mathbf{F i g . 2 e}$ ).

\section{Results}

\section{Clinical characteristics and the therapeutic outcomes}

Patient and lesion characteristics are shown in $>$ Table 1. Median age of the 127 patients was 56 years (range, $28-80$ ), and the proportions of male and female patients were $58.3 \%$ and $41.7 \%$, respectively. Gross types of lesions were as follows: seven patients (5.5\%) were type 0 -Ila, 26 (20.5\%) were type 0 -IIb, and 94 (74.0\%) were type 0 -Ilc. Lesions were located in the lower, middle, and upper third in 40 patients (31.5\%), 86 (67.7\%), and $1(0.8 \%)$, respectively. Circumferential locations were as follows: the anterior wall in 25 patients (19.7\%), posterior wall in $27(21.2 \%)$, lesser curvature in 38 (29.9\%), and greater curvature in 27 (21.2\%). Median tumor size during the preoperative diagnosis was $13 \mathrm{~mm}$ (range, 3-20). Number of circumferential biopsies were as follows: four in 110 patients (86.6\%); five in 13 (10.2\%); and six in 4 (3.2\%). There were four patients (3.2\%) in whom circumferential biopsies were cancer-positive; secondary preoperative EGDs were carried out to confirm whether the additional circumferential biopsies were negative in all patients.

Therapeutic outcomes of ESD are shown in > Table 2. Median size of ESD specimens was $35 \mathrm{~mm}$ (range, 18-56). Median tumor size was $12 \mathrm{~mm}$ (range, 1-29). Median operative time was 70 minutes (range, 25-265). The histologically dominant type was SRC, which was diagnosed in 110 patients (86.6\%), followed by PDAC in 17 (13.4\%). The rate of en-bloc resection was $100 \%(127 / 127)$, whereas those of R0 and curative resections were $97.6 \%(124 / 127)$ and $80.3 \%$ (102/127), respectively. Non-curative resection was pathologically identified in 25 pa- tients (19.7\%) overall. The reasons for incurability were as follows: tumor size $>20 \mathrm{~mm}$ in 18 patients (14.2\%), submucosal invasion in eight $(6.3 \%)$, ulcer findings in four $(3.2 \%)$, lymphovascular invasion in three $(2.4 \%)$, and $\mathrm{LM}+(\mathrm{LM}+:<2 \mathrm{~mm}$ to the lateral margin) in three (2.4\%). Three patients $(2.4 \%)$ had delayed bleeding, and none had perforation. None of the patients had local or metastatic recurrences. The median observation period was 27 months (range, 1-50). Circumferential biopsy has successfully identified the misdiagnosis of cancerous areas of four patients $(3.2 \%)$, with three $(2.4 \%)$ achieving LM-resection.

The overall median shortest distance was $7 \mathrm{~mm}$ (range, 0 13). In 112 patients ( $88.2 \%$ ), the shortest distance was $\geq 5 \mathrm{~mm}$, which was considered as a safe LM. In 15 patients (11.8\%), the shortest distance was $<5 \mathrm{~mm}$, which was considered as an insufficient LM ( $\triangleright$ Fig. 3 ).

\section{Clinical characteristics of patients with LM +}

Characteristics of three patients $(2.4 \%)$ with $\mathrm{LM}+$, which was pathologically identified, are shown in $>$ Table 3. All LM+patients had non-indication lesions for ESD, such as a those with a tumor size $>20 \mathrm{~mm}$. In all $\mathrm{LM}+$ patients, the cancerous area had spread within the atrophic mucosa in the lesser curvature of the stomach from the angle to the lower gastric body. Two out of three $\mathrm{LM}+$ patients underwent additional treatments. One patient underwent secondary ESD 3 weeks after initial ESD, and the residual carcinoma, which was close to the ESD scar, was successfully resected. Another patient underwent laparoscopic distal gastrectomy, and a $7 \times 7-\mathrm{mm}$ residual carcinoma without lymph node metastasis was found in the oral side of the post-ESD scar ( $\triangleright$ Fig.4). However, another elderly patient refused to undergo additional treatment due to his age ( $>70$ years) and chronic heart failure. This patient was followed up for 38 months, and no recurrence has been observed to date. 
- Table 2 Therapeutic outcomes of 127 patients who underwent endoscopic submucosal dissection for undifferentiated-type early gastric cancer (UD-type EGC).

\begin{tabular}{|c|c|}
\hline & UD-type EGC $(n=127)$ \\
\hline Specimen size, median (range), mm & $35(18-56)$ \\
\hline Tumor size (pathological diagnosis), median (range), mm & $12(1-29)$ \\
\hline Operative time, median (range), minute & $70(25-265)$ \\
\hline Histology (dominant type), n (\%) SRC / PDAC & $110(86.6) / 17(13.4)$ \\
\hline En bloc resection, $\mathrm{n}(\%)$ & $127(100)$ \\
\hline R0 resection, $\mathrm{n}(\%)$ & $124(97.6)$ \\
\hline Curative resection, $\mathrm{n}(\%)$ & $102(80.3)$ \\
\hline \multicolumn{2}{|l|}{ Factors for non-curative resection, $\mathrm{n}(\%)^{1}$} \\
\hline - Tumor diameter > 20 mm & $18(14.2)$ \\
\hline - Submucosal invasion & $8(6.3)$ \\
\hline - Ulcer findings & $4(3.2)$ \\
\hline - Lymphatic-vascular involvement & $3(2.4)$ \\
\hline - Lateral margin positive & $3(2.4)$ \\
\hline \multicolumn{2}{|l|}{ Complications, n (\%) } \\
\hline - Delayed bleeding & $3(2.4)$ \\
\hline - Perforation & $0(0)$ \\
\hline Local and metastatic recurrence, $\mathrm{n}(\%)$ & $0(0)$ \\
\hline Observation period, median (range), month & $27(1-50)$ \\
\hline The shortest distance, median (range), mm & $7(0-13)$ \\
\hline \multicolumn{2}{|c|}{$\begin{array}{l}\text { The shortest distance, The shortest distance from the lesion edge to the specimen edge. } \\
\text { SRC, signet ring cell carcinoma; PDAC, poorly differentiated adenocarcinoma } \\
{ }^{1} \text { Some patients had more than one curative factor. }\end{array}$} \\
\hline
\end{tabular}

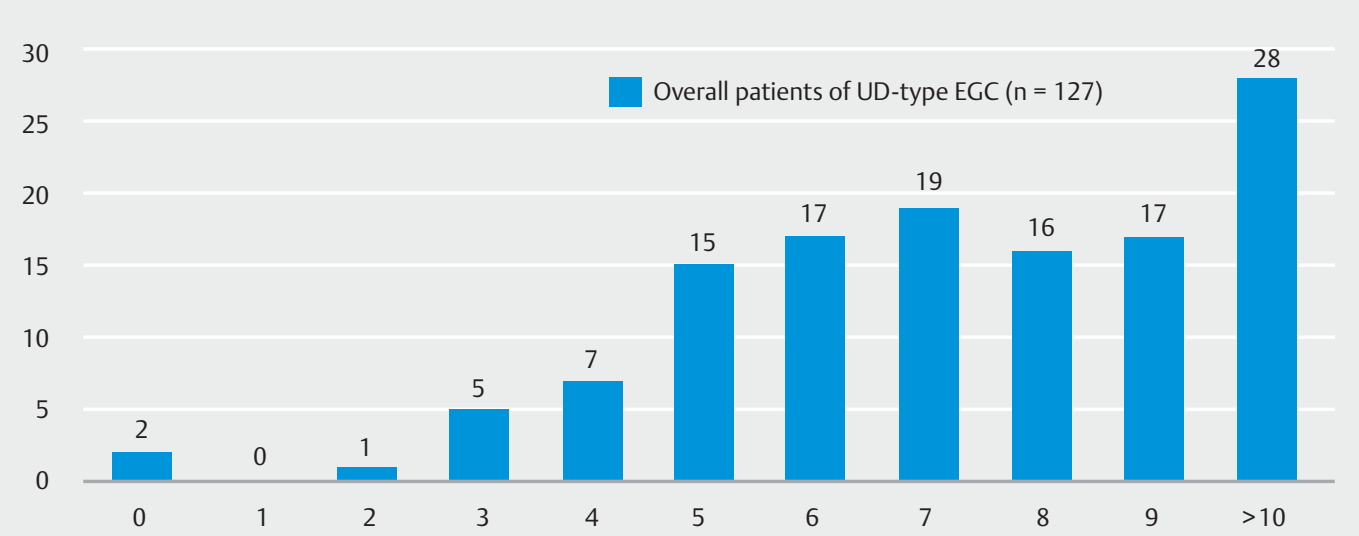

- Fig. 3 Distribution of the shortest distance from the lesion edge to the specimen edge in all patients. Median shortest distance is $7 \mathrm{~mm}$ (range, 0 - 13). Furthermore, 112 patients $(88.2 \%$ ) had the shortest distance of $\geq 5 \mathrm{~mm}$, which is considered the safety lateral margin. Three patients (2.4\%) were diagnosed as lateral margin positive.

\section{Discussion}

Generally, the LM-resection rate in ESD for D-type EGC was reported as $96.9 \%$ to $99.0 \%$ [10 - 12], whereas that for UD-type EGC was reported as $72.7 \%$ to $94.8 \%$ [5-9], which is lower than the result of D-type EGC. Our marking method for UDtype EGC, which is securing the LM of approximately $5 \mathrm{~mm}$ from the estimated border of lesion after the M-NBI diagnosis, and circumferential biopsies achieved a low LM-resection rate 
- Table 3 Clinical characteristics of undifferentiated-type early gastric cancer with pathologically positive lateral margin.

\begin{tabular}{|c|c|c|c|c|c|c|c|c|c|}
\hline Case & $\begin{array}{l}\text { Agel } \\
\text { Sex }\end{array}$ & Location & $\begin{array}{l}\text { Number of } \\
\text { circumferential } \\
\text { biopsies }\end{array}$ & $\begin{array}{l}\text { Tumor } \\
\text { size } \\
(\mathrm{mm})\end{array}$ & $\begin{array}{l}\text { Specimen } \\
\text { size } \\
(\mathrm{mm})\end{array}$ & $\begin{array}{l}\text { Histol- } \\
\text { ogy }\end{array}$ & $\begin{array}{l}\text { Inva- } \\
\text { sion } \\
\text { depth }\end{array}$ & $\begin{array}{l}\text { The shortest } \\
\text { distance } \\
(\mathrm{mm})\end{array}$ & $\begin{array}{l}\text { Additional } \\
\text { therapy }\end{array}$ \\
\hline 1. & $65 / M$ & $\begin{array}{l}\text { Lesser curvature } \\
\text { of angle }\end{array}$ & 4 & 21 & 30 & SRC & M & 0 & Re-ESD \\
\hline 2. & $64 / M$ & $\begin{array}{l}\text { Lesser curvature } \\
\text { of lower body }\end{array}$ & 5 & 25 & 47 & SRC & M & 0 & $\begin{array}{l}\text { Distal } \\
\text { gastrect- } \\
\text { omy }\end{array}$ \\
\hline 3. & $73 / \mathrm{M}$ & $\begin{array}{l}\text { Lesser curvature } \\
\text { of lower body }\end{array}$ & 4 & 25 & 35 & SRC & M & 2 & No \\
\hline
\end{tabular}

The shortest distance, The shortest distance from the lesion edge to the specimen edge.

SRC, signet ring cell carcinoma; ESD, endoscopic submucosal dissection
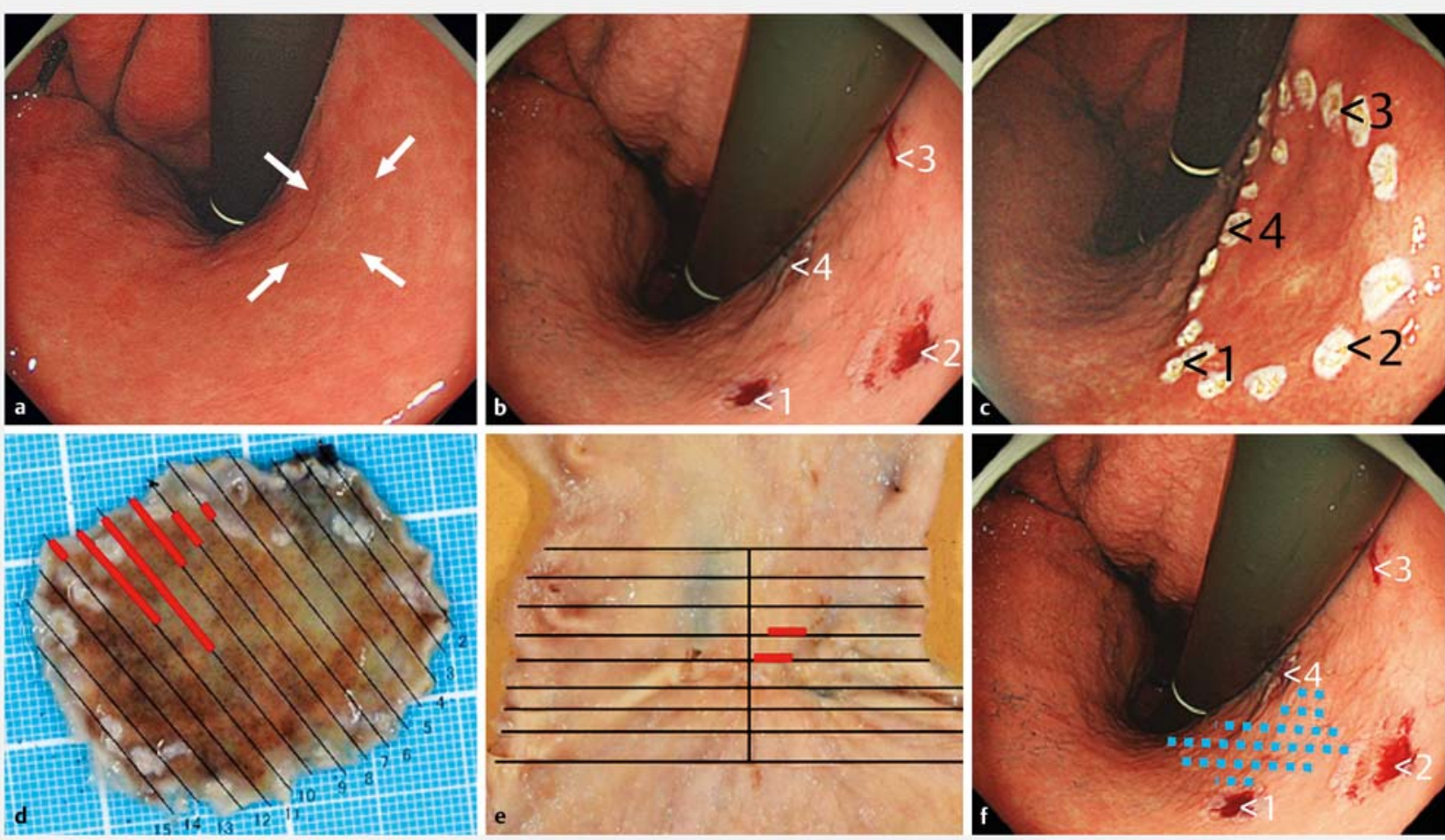

- Fig. 4 A case of undifferentiated-type early gastric cancer with pathologically positive lateral margin in a 64-year-old man. a In preoperative esophagogastroduodenoscopy, a discolored lesion is located on the lesser curvature of the lower gastric body (white arrow). b A diagnostic demarcation using $\mathrm{M}-\mathrm{NBI}$ is performed and then, four circumferential biopsies (numbers 1 to 4 ) are obtained $5 \mathrm{~mm}$ apart from the lesion, which were identified as cancer-negative samples. $\mathbf{c}$ The markings are placed on the circumferential biopsy scars and ESD is performed.

d Histological mapping: the ESD specimen is excised as indicated by the black lines. The area of the lesion itself is represented by the red lines. LM is positive in four slices. Pathological findings: ESD specimen size, $43 \times 35 \mathrm{~mm}$; tumor size, $18 \times 16 \mathrm{~mm}$; and type 0 -Ilc, Signet ring cell carcinoma, UL-, M, ly0, v0, LM +, VM-. e Laparoscopic distal gastrectomy is performed as an additional therapy, and $7 \times 7$-mm residual carcinoma is observed in the oral side of post-ESD scar. $\mathbf{f}$ On retrospective consideration, the area of the lesion itself is depicted by the blue dotted line. We did not realize the misdiagnosis of the cancerous areas because the lesion is spread between the circumferential biopsy sites (numbers 1 to 4 ). $\mathrm{M}-\mathrm{NBI}$, magnifying endoscopy with narrow-band imaging; ESD, endoscopic submucosal dissection; LM, lateral margin; VM, vertical margin.

(97.6\%), which is an excellent result compared to the previous reports. Hwang et al. reported that the residual/recurrent tumor rate was $34.5 \%$ in cases with pathologically diagnosed LM + resection, and undifferentiated histology was an independent risk factor for the development of residual/recurrent tumors
[15]. Therefore, the standard secondary management in cases with $\mathrm{LM}+$ resection for UD-type EGC is surgical gastrectomy [3], and LM-resection is important.

Following recent advances in M-NBI, our institution previously reported [13] the by-growth pattern on the M-NBI fea- 

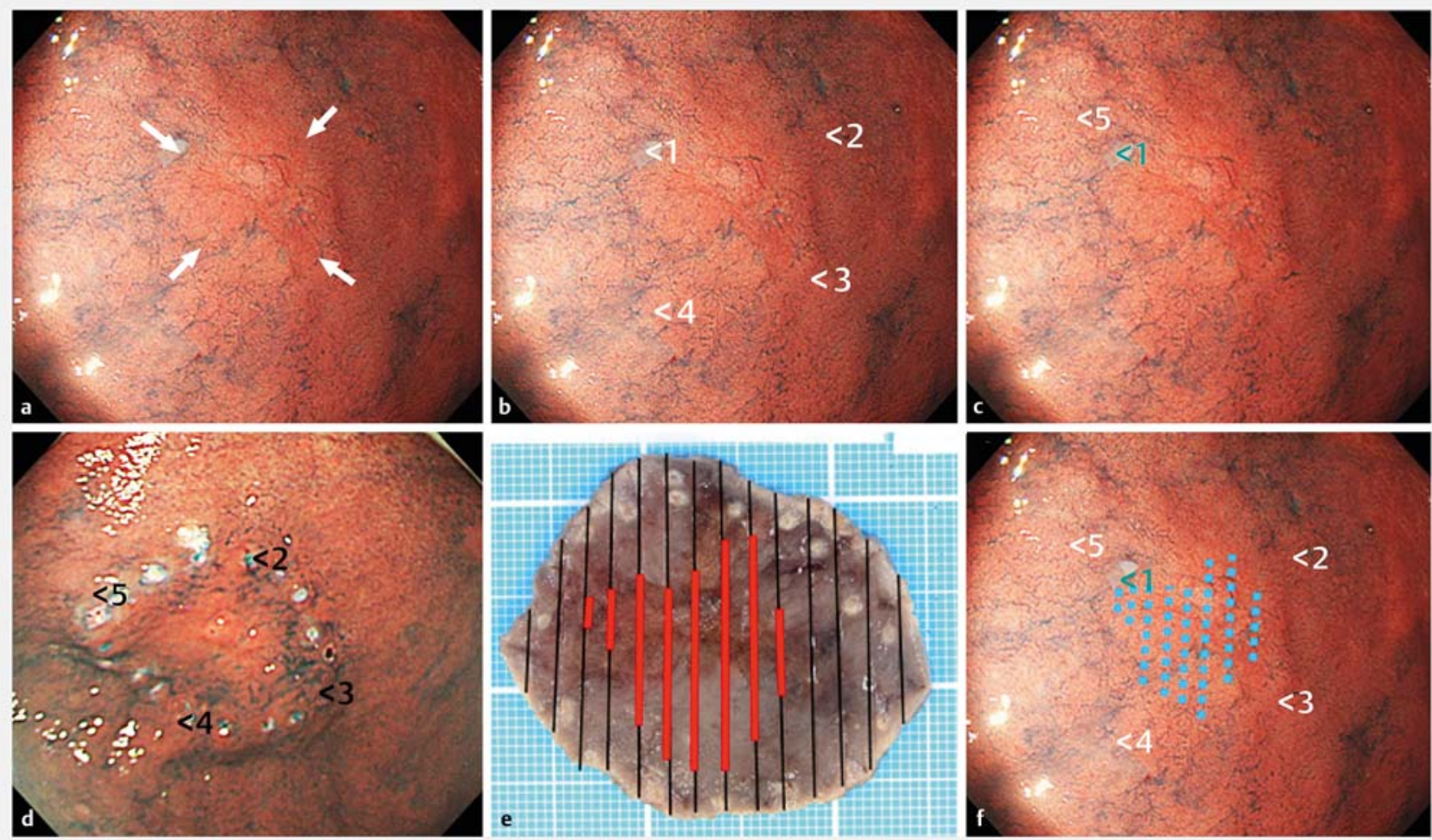

- Fig. 5 A case of undifferentiated type early gastric cancer with cancer-positive circumferential biopsy results. a In preoperative esophagogastroduodenoscopy (EGD), a discolored lesion is located on the anterior wall of the lower gastric body (white arrow). b A diagnostic demarcation using M-NBI is performed and then, four circumferential biopsies (numbers 1 to 4 ) are obtained 5 mm apart from the lesion. Numbers 2 to 4 biopsies are identified as cancer-negative samples, whereas the number 1 biopsy is identified as a cancer-positive sample. $\mathrm{c}$ In secondary preoperative EGD, one additional circumferential biopsy (number 5 ) is obtained approximately $3 \mathrm{~mm}$ outside from the lesion, which is identified as cancer-negative. $\mathbf{d}$ The markings are placed on the circumferential biopsy scars (numbers 2 to 5 ) and ESD is performed. e Pathological findings: ESD specimen size, $46 \times 40 \mathrm{~mm}$; tumor size, $24 \times 23 \mathrm{~mm}$; and type 0 -Ilc, poorly differentiated adenocarcinoma, UL-, M, ly0, v0, LM-, VM-. $\mathbf{f}$ On retrospective consideration, the area of the lesion itself is depicted by the blue-dotted line. Number 1 biopsy is located on the edge of the lesion. By placing the markings on the number 5 biopsy scar, LM- resection is performed successfully. M-NBI, magnifying endoscopy with narrow-band imaging; ESD, endoscopic submucosal dissection; LM, lateral margin; VM, vertical margin.

tures of UD-type EGC and identified and compared significant expansion of the intervening parts within neoplastic lesions with noncancerous regions. Expansion of the intervening parts enhanced the diagnostic capability, and accurate diagnosis rates for the diagnostic demarcation of UD-type EGC improved from $53.9 \%$ to $81.5 \%$ with the addition of M-NBI to WLI [16]. Thus, accurate identification of the cancerous areas has become possible even in UD-type EGC. In other procedures for precise diagnostic delineation of cancerous areas, circumferential biopsies for confirming the noncancerous areas are useful to identify a misdiagnosis of the cancerous areas. Cancerous areas of lesions with lateral extension beneath the noncancerous mucosa, which is at a high risk of LM + resection by ESD [4, 11], are sometimes difficult to precisely and diagnostically delineate, even when using M-NBI. In particular, achieving LM-resection in such lesions necessitates the consideration of circumferential biopsies on preoperative EGD. In this study, four patients (3.2\%) were cancer-positive by circumferential biopsy; of these, three achieved LM-resection by undergoing second- ary preoperative EGD to confirm whether the additional circumferential biopsies were negative ( $\bullet$ Fig. 5 ).

In this study, the cancerous area in three patients with LM + resection had spread under the atrophic mucosa in the lesser curvature of the stomach from the angle to the lower body. Diagnostic demarcation in the lesser curvature of the stomach between the angle and gastric body is difficult due to the strong atrophic changes in the background mucosa and endoscopic observation from a tangential angle. Moreover, in those patients with $L M+$ resection, circumferential biopsies could not identify the misdiagnosis of cancerous areas, because the cancerous area had spread between the sites of the circumferential biopsies ( $\triangleright$ Fig. 4 ). Although larger resection is a simple strategy for LM-resection, unnecessary larger resection causes more bleeding as more vessels would be exposed in the base of the ulcers after ESD. Several studies have reported that ESD specimen size of $>40 \mathrm{~mm}$ was a significant risk factor for post-ESD bleeding [17-19]. In this study, $2.3 \%$ of patients had post-ESD bleeding, which is lower than the rates in previous studies. This could be due to the fact that the median size of the ESD speci- 
mens was $35 \mathrm{~mm}$ in this study, which was $<40 \mathrm{~mm}$. The minimum required specimen size of ESD may have contributed to the low post-ESD bleeding rate.

The current study had some limitations. First, this was a nonrandomized, retrospective, and single-center study. Second, this study included a small number of patients. Third, a bias of patient selection might have been present. To reduce the selection bias, we included consecutive patients except for those who satisfied the exclusion criteria. However, a future prospective, randomized, multicenter study is needed to confirm with certainty the benefits of the marking methods for LM-resection in ESD for UD-type EGC.

\section{Conclusion}

In conclusion, our marking method, which secures an LM of approximately $5 \mathrm{~mm}$ after $\mathrm{M}-\mathrm{NBI}$ diagnosis and four or more circumferential biopsies, may reduce $L M+$ resections in ESD for UD-type EGC.

\section{Competing interests}

None

\section{References}

[1] Leung WK, Wu MS, Kakugawa Y et al. Screening for gastric cancer in Asia: current evidence and practice. Lancet Oncol 2008; 9: 279-287

[2] Hirasawa T, Gotoda T, Miyata S et al. Incidence of lymph node metastasis and the feasibility of endoscopic resection for undifferentiated type early gastric cancer. Gastric Cancer 2009; 12: 148-152

[3] Japanese Gastric Cancer Association. Japanese gastric cancer treatment guidelines 2010 (ver. 3). Gastric Cancer 2011; 14: 113-123

[4] Ninomiya A, Yanagisawa Y, Kato Y et al. Unrecognizable intramucosal spread of diffuse-type mucosal gastric carcinoma of less than $20 \mathrm{~mm}$ in size. Endoscopy 2000; 32: 604-608

[5] Yamamoto Y, Fujisaki J, Hirasawa T et al. Therapeutic outcomes of endoscopic submucosal dissection of undifferentiated-type intramucosal gastric cancer without ulceration and preoperatively diagnosed as 20 millimeters or less in size. Dig Endosc 2010; 22: 112-118
[6] Abe S, Oda I, Suzuki H et al. Short- and long-term outcomes of endoscopic submucosal dissection for undifferentiated early gastric cancer. Endoscopy 2013; 45: $703-707$

[7] Oka S, Tanaka S, Higashiyama M et al. Clinical validity of the expanded criteria for endoscopic resection of undifferentiated-type early gastric cancer based on long-term outcomes. Surg Endosc 2014; 28 : 639-647

[8] Park JC, Lee YK, Kim SY et al. Long-term outcomes of endoscopic submucosal dissection in comparison to surgery in undifferentiatedtype intramucosal gastric cancer using propensity score analysis. Surg Endosc 2018; 32: 2046-2057

[9] Jeon HK, Lee S], Kim GH et al. Endoscopic submucosal dissection for undifferentiated-type early gastric cancer: short- and long-term outcomes. Surg Endosc 2018; 32: $1963-1970$

[10] Ono H, Hasuike N, Inui T et al. Usefulness of a novel electrosurgical knife, the insulation-tipped diathermic knife-2, for endoscopic submucosal dissection of early gastric cancer. Gastric Cancer 2008; 11 : $47-52$

[11] Kakushima N, Ono H, Tanaka M et al. Factors related to lateral margin positivity for cancer in gastric specimens of endoscopic submucosal dissection. Dig Endosc 2011; 23: 227-232

[12] Min BH, Kim KM, Park CK et al. Outcomes of endoscopic submucosal dissection for differentiated-type early gastric cancer with histological heterogeneity. Gastric Cancer 2015; 18: 618-626

[13] Okada K, Fujisaki J, Kasuga A et al. Diagnosis of undifferentiated-type early gastric cancers by magnification endoscopy with narrow-band imaging. J Gastroenterol Hepatol 2011; 26: 1262 -1269

[14] Japanese GastricCancer Association. Japanese classification of gastric carcinoma: 3rd English edition. Gastric Cancer 2011; 14: 101 - 112

[15] Hwang J], Park KJ, Park YS et al. A scoring system for patients with a tumor-positive lateral resection margin after endoscopic resection of early gastric cancer. Surg Endosc 2016; 30: 2751-2758

[16] Horiuchi Y, Fujisaki J, Yamamoto $N$ et al. Accuracy of diagnostic demarcation of undifferentiated-type early gastric cancers for magnifying endoscopy with narrow-band imaging: endoscopic submucosal dissection cases. Gastric Cancer 2016; 19: 515-523

[17] Okada K, Yamamoto Y, Kasuga A et al. Risk factors for delayed bleeding after endoscopic submucosal dissection for gastric neoplasm. Surg Endosc 2011; 25: 98-107

[18] Kataoka Y, Tsuji Y, Sakaguchi Y et al. Bleeding after endoscopic submucosal dissection: Risk factors and preventive methods. World ] Gastroenterol 2016; 22: 5927-5935

[19] Koh R, Hirasawa K, Yahara S et al. Antithrombotic drugs are risk factors for delayed postoperative bleeding after endoscopic submucosal dissection for gastric neoplasms. Gastrointest Endosc 2013; 78: 476 483 